OPEN ACCESS

Edited by:

Juan Andrés Orellana Pontificia Universidad Católica de

Chile, Chile

Reviewed by:

Maite A. Castro,

Universidad Austral de Chile, Chile

Juan C. Saez,

Pontificia Universidad Católica de

Chile, Chile

*Correspondence:

Sonia L. Albarracín albarra@javeriana.edu.co

Received: 16 November 2017 Accepted: 27 March 2018 Published: 11 April 2018

Citation:

Mayorquin LC, Rodriguez AV, Sutachan J-J and Albarracín SL (2018) Connexin-Mediated Functional and Metabolic Coupling Between Astrocytes and Neurons.

Front. Mol. Neurosci. 11:118. doi: 10.3389/fnmol.2018.00118

\section{Connexin-Mediated Functional and Metabolic Coupling Between Astrocytes and Neurons}

\author{
Lady C. Mayorquin, Andrea V. Rodriguez, Jhon-Jairo Sutachan and Sonia L. Albarracín* \\ Departamento de Nutrición y Bioquímica, Facultad de Ciencias, Pontificia Universidad Javeriana, Bogotá, Colombia
}

The central nervous system (CNS) requires sophisticated regulation of neuronal activity. This modulation is partly accomplished by non-neuronal cells, characterized by the presence of transmembrane gap junctions (GJs) and hemichannels (HCs). This allows small molecule diffusion to guarantee neuronal synaptic activity and plasticity. Astrocytes are metabolically and functionally coupled to neurons by the uptake, binding and recycling of neurotransmitters. In addition, astrocytes release metabolites, such as glutamate, glutamine, D-serine, adenosine triphosphate (ATP) and lactate, regulating synaptic activity and plasticity by pre- and postsynaptic mechanisms. Uncoupling neuroglial communication leads to alterations in synaptic transmission that can be detrimental to neuronal circuit function and behavior. Therefore, understanding the pathways and mechanisms involved in this intercellular communication is fundamental for the search of new targets that can be used for several neurological disease treatments. This review will focus on molecular mechanisms mediating physiological and pathological coupling between astrocytes and neurons through GJs and HCs.

Keywords: gliotransmission, connexins, synaptic plasticity, neurons, astrocytes, neurodegenerative disease, hypoxia, ischemia

\section{INTRODUCTION}

Classical studies carried out by the end of 19th and during the 20th century classified astrocytes mainly based on their differences in morphology and localization into two different populations, protoplasmic and fibrous (Ramón y Cajal, 1909). However, recent studies have revealed a more intricate and complex level of astrocyte organization in the central nervous system (CNS). Specialized astrocytes and stem cells with astrocyte features have been found in the cerebellum and retina (Bergmann glia and Müller cells) and neurogenic niches (Ben Haim and Rowitch, 2017). Likewise, these astrocyte populations are characterized by different marker expression. Astrocytes located in the white matter and astrocytic stem cells express high levels of glial fibrillary acid protein (GFAP). In contrast, protoplasmic astrocytes exhibit low expression of GFAP but high levels of the potassium channel Kir4.1 (Ben Haim and Rowitch, 2017). Although, it remains unclear why astrocytes achieve such diversity; this could be an adaptive mechanism to the activity of specific neuronal networks.

Due to their intimate association with neurons and blood vessels cells, astrocytes play a metabolic, structural and regulatory role in the CNS. For instance, astrocytes supply energetic substrates (glucose, lactate, citrate, and glutamine) to neurons, participate in the turnover of neurotransmitters such as glutamate and gamma-aminobutyric acid (GABA; Barres, 2008; Sofroniew and Vinters, 2010), and regulate the blood-brain barrier (BBB) permeability, and the homeostasis of ions and pH (De Bock et al., 2017). In addition, astrocytes can release different growth factors that regulate synapse formation and expression of tight junction proteins (Liebner et al., 2011). 
Complex astrocyte endeavors are in part explained by their intercellular communications through gap junctions (GJs). This specialized intercellular communication gives rise to a complex syncytial network that allows diffusion of several essential molecules for signaling and information processing (Robertson, 2013). GJs and hemichannels (HCs) formed by connexins (Cxs) allow small molecule diffusion that induces and regulate functional and metabolic coupling between astrocytes and neurons. Neurotransmitters released by neurons bind to astrocyte membrane-bound receptors, resulting in the production of metabolites, such as inositol 1,4,5 trisphosphate $\left(\mathrm{IP}_{3}\right)$, adenosine triphosphate (ATP), glutamate, lactate and D-serine that diffuse through GJs to impact back on neuronal function (Orellana and Stehberg, 2014). As a case in point, in the gray matter, it has been shown individual protoplasmic astrocytes occupying non-overlapping spatial domains, where each astrocyte can interact with hundreds of dendrites and neuronal cell bodies (Oberheim et al., 2009; Sofroniew and Vinters, 2010). This bidirectional interaction is fundamental for healthy brain function, and impairment in this communication can lead to the development of pathological conditions. This review will focus on cellular and molecular mechanisms that mediate metabolic and functional coupling between astrocytes and neurons, and how Cxs-mediated intercellular communication in astrocytes play a crucial role in this coupling. Additionally, we will review our current understanding of the neuroglial interaction emerging role in some neurological disease development.

\section{CONNEXIN EXPRESSION IN THE CNS}

Cxs are a family of transmembrane proteins composed of six subunits forming homomeric or heteromeric HCs that participate in small molecule release (up to $1.5 \mathrm{kDa}$ ) directly to the extracellular space. HCs also dock with other HCs or connexons of adjacent cell membranes to establish GJs (White and Bruzzone, 1996; Figure 1). At the structural level, Cxs have four transmembrane domains and one intracellular domain involved in channel regulation by post-translational modifications (i.e., phosphorylations) or interactions with cytosolic proteins (Márquez-Rosado et al., 2012; Matsuuchi and Naus, 2013; Figure 1). Of the $21 \mathrm{Cxs}$ isoforms so far described, 11 have been detected in the CNS. Cxs in astrocytes, oligodendrocytes, microglia and neurons are characterized by developmental state, region and cell-type specific isoform expression, suggesting a critical role of these proteins in regulation and maintenance of several CNS functions (Lapato and Tiwari-Woodruff, 2018).

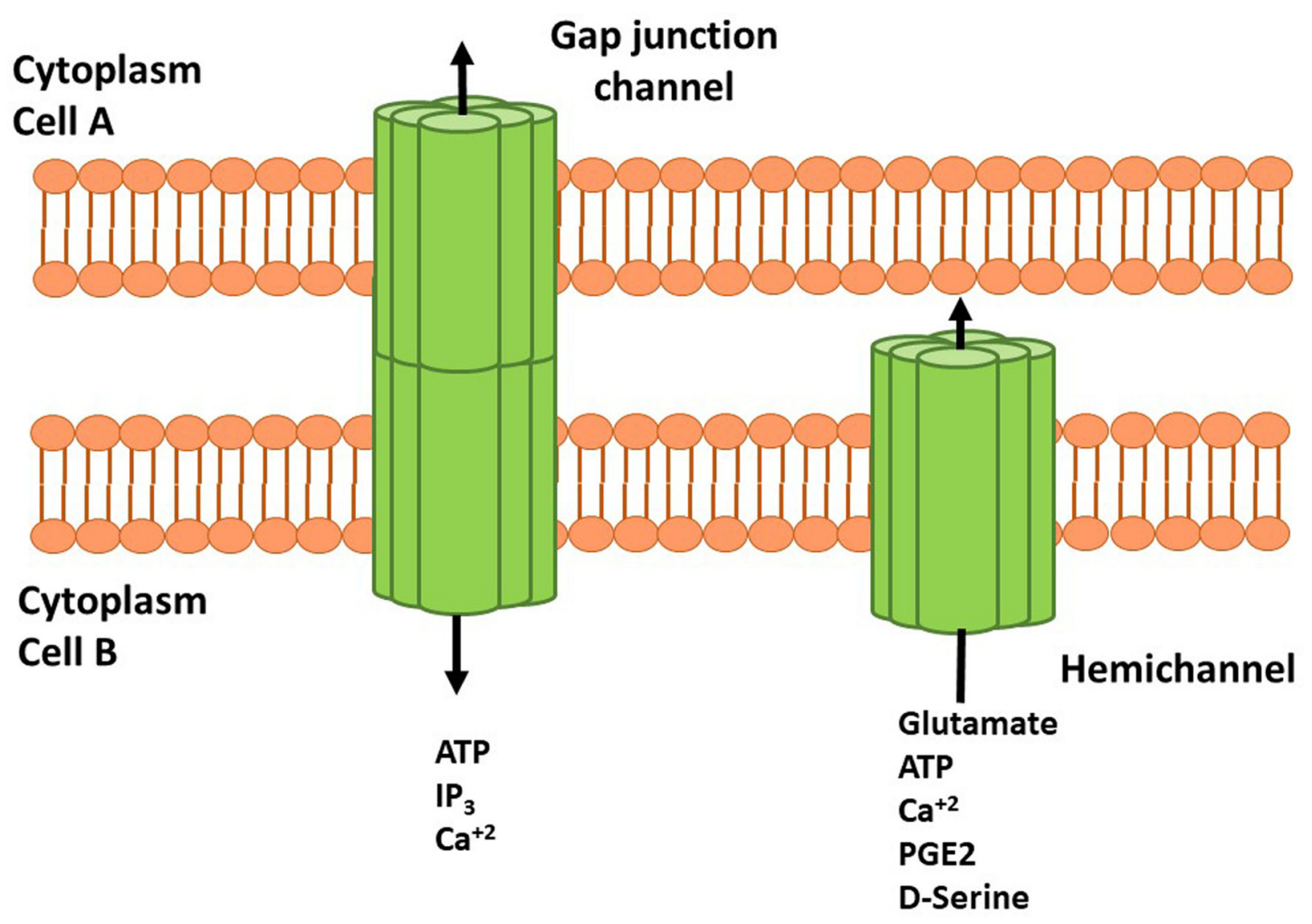

FIGURE 1 | Schematic representation of connexins (CXs), hemichannels (HCs) and gap junctions (GJs) in cell membranes. HCs are transmembrane proteins composed of six connexin subunits that allow flow of several molecules and gliotransmitters from astrocyte to the extracellular space. Adenosine Triphosphate (ATP), glutamate, D-serine and prostaglandin (PG) E-2 can interact with their receptors and induce signaling cascades. Docking between two HCs or connexins forms GJ channels that allow the cell to cell communication mediated by ATP, inositol 1,4,5 trisphosphate (IP3) and Ca ${ }^{2+}$. HCs and GJs activity modulate neuronal synaptic activity and plasticity. 
Cxs not only allow ions, small metabolites, or second messengers flow but also mediate or regulate other processes in the CNS. For example, $\mathrm{Cx} 43$ controls migration and positioning of excitatory and cortical interneurons during brain development (Elias et al., 2010; Qi et al., 2016). Cxs are also involved in neurogenesis; while $\mathrm{Cx} 43$ promotes survival of newborn neurons in the adult mouse hippocampus; Cx30 limits their proliferation and survival (Liebmann et al., 2013). Magnotti et al. (2011) have reported Cx42 and Cx43 are required for astrocyte survival in white matter by mechanisms dependent on Cxs specific expression in both astrocytes and oligodendrocytes. Therefore, differential expression and spatial distribution of Cxs are important determinants of their functions in the CNS.

In the mature brain, astrocytes express high levels of Cx30 and Cx43 and low Cx26 levels. Possibly this expression profile is responsible for determining the autocrine and paracrine signaling interaction that mediates glial and neuroglial communications (Lapato et al., 2017). GJs in astrocytes facilitate the formation of a functional syncytium, promoting ion and neurotransmitter removal released during neuronal activity, and allow propagation of $\mathrm{Ca}^{2+}$ waves between astrocytes (Takeuchi and Suzumura, 2014). This interglial communication through Cx-based channels, allows astrocytes to sense and integrate local and global synaptic activity and to respond to gliotransmitters that impact synaptic transmission both pre- and postsynaptically (Bazargani and Attwell, 2016).
Hence, Cxs and gliotransmitters are essential players in neuronal activity regulation, behavior, and homeostatic maintenance of brain functions (Volterra and Meldolesi, 2005).

\section{CONNEXIN-DEPENDENT REGULATION OF SYNAPTIC TRANSMISSION AND PLASTICITY}

Astrocytes are unable to generate action potentials; however, they can raise intracellular calcium concentrations $\left(\left[\mathrm{Ca}^{2+}\right] \mathrm{i}\right)$ that spread from cell to cell. Presence of astrocytic calcium ions mobilizations suggests that glial cells may have some excitability and neuromodulator activities (Bezzi and Volterra, 2001; Volterra and Meldolesi, 2005; Dallérac et al., 2013). During intense neuronal firing, glutamate and GABA release induce elevations in glial cells $\left[\mathrm{Ca}^{2+}\right] \mathrm{i}$ (Cornell-Bell et al., 1990; Kang et al., 1998). This in turn causes a $\mathrm{Ca}^{2+}$-dependent release of molecules that impact on neuronal excitability and synaptic transmission and plasticity (Bazargani and Attwell, 2016). Molecules released by astrocytes upon $\left[\mathrm{Ca}^{2+}\right] \mathrm{i}$ increase led to gliotransmitters discovery, which regulates communication between astrocytes and neurons. Hence, it is now accepted that astrocytes work along with neurons through a feedback mechanism, in which neuronal activity induces astrocytes to regulate synaptic transmission and plasticity by releasing gliotransmitters (Figure 2).

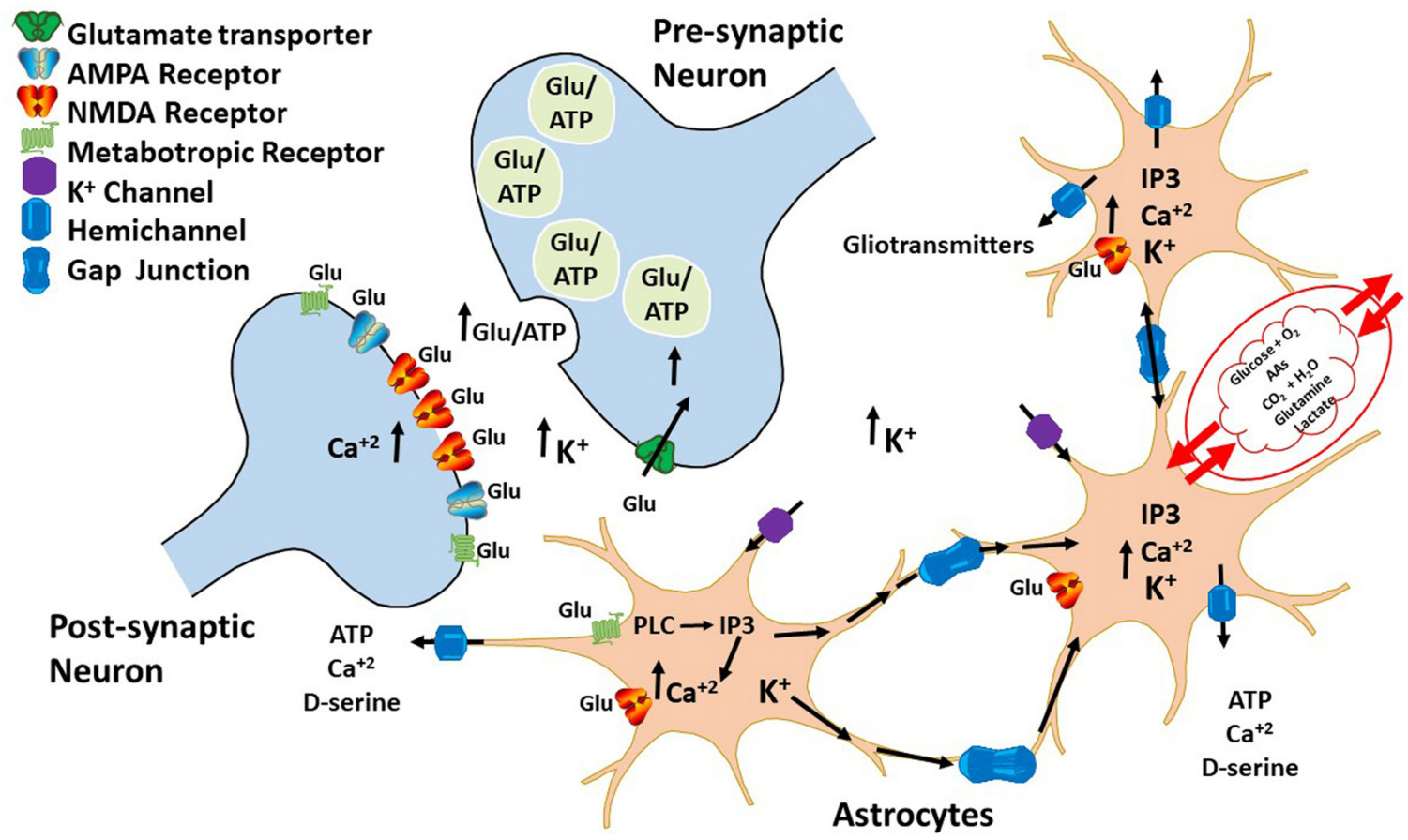

FIGURE 2 | Bidirectional communication between astrocytes and neurons. During neuronal firing, neurons release neurotransmitters that bind to G-coupled receptors expressed on the surface of astrocytes. The activation of these receptors leads to an increase in $\mathrm{IP}_{3}$ and intracellular calcium ions that diffuses through HCs to other astrocytes, inducing gliotransmitter release, such as ATP and glutamate. These gliotransmitters then regulate both glutamatergic and GABAergic neurotransmission by acting either pre- or post-synaptically. 
According to this paradigm neurons convey information to astrocytes mostly through neurotransmitter release. In this sense, different studies have shown hippocampal CA1 stratum radiatum astrocytes respond with an increase in $\left[\mathrm{Ca}^{2+}\right] \mathrm{i}$ when activated by ligands. Among the many receptors expressed in astroglial cells are purinergic (Duffy and MacVicar, 1995; Pasti et al., 1997; Porter and McCarthy, 1997; Verkhratsky et al., 1998; Shelton and McCarthy, 1999, 2000; Bowser and Khakh, 2007; Fiacco et al., 2007; Navarrete and Araque, 2008), adrenergic (Duffy and MacVicar, 1995), glutamatergic (Shelton and McCarthy, 1999; Fiacco et al., 2007), and GABAergic. In vitro studies have shown glutamate-induced astrocyte $\left[\mathrm{Ca}^{2+}\right] \mathrm{i}$ increase through ionotropic (NMDA and AMPA) and metabotropic glutamate receptor (mGLURs) activation (Zhang et al., 2003; Hu et al., 2004). Interestingly, in vivo experiments have revealed this $\left[\mathrm{Ca}^{2+}\right]$ i elevation occurs only in some regions of the brain cortex (Pasti et al., 1997; Volterra et al., 2014). A mechanism illustrating in vivo cortical astrocyte differential response to glutamate has not been fully established. However, it is thought that some regulatory mechanisms may be conditioning the response of these astrocytes. A recent study revealed hippocampal astrocytes discriminate between neurotransmitters released by different axonal pathways (Perea and Araque, 2005). CA1 astrocytes in the hippocampus respond to glutamate released from CA3 neurons (Schaffer collateral, SC) and to acetylcholine, but not to glutamate released by CA1 neurons (Alveus Terminalis, AT). A plausible explanation for this phenomenon is glutamate protein sensors (receptors and transporters) are differentially localized in these astrocytes. Hence, CA1 astrocytes respond to glutamate released by SC axons, because sensors are expressed in astrocytic processes that are close to these fibers. Nevertheless, the fact that a same population of astrocytes can respond specifically to different neurotransmitters, also suggests astrocytes have intrinsic properties that allow them to discriminate and integrate synapse activity (Perea and Araque, 2005).

Neurotransmitters mainly impact astrocytic $\left[\mathrm{Ca}^{2+}\right]_{i}$ by receptor activation. However, it has also been shown astrocytes express transporters that also regulate synaptic transmission through neurotransmitter uptake (Boddum et al., 2016). GABA is the primary inhibitory neurotransmitter in the brain. It exerts its function by either stimulating chloride ions influx or potassium ions efflux by activating ionotropic and metabotropic GABA receptors (Watanabe et al., 2002). Astrocytes express the GABA transporter GAT-3, whose function is to clear excessive GABA from the synaptic cleft (Ribak et al., 1996). A recent finding suggests GABA uptake throughout GAT-3 can cause inhibition of presynaptic neuronal glutamate release by ATP/adenosine released from astrocytes (Boddum et al., 2016). Different to the canonical pathway in which neurotransmitters directly induce a rise in $\left[\mathrm{Ca}^{2+}\right] \mathrm{i}$, GABA increases astrocyte excitability by movement of sodium ions to the intracellular milieu, resulting in calcium ion influx through $\mathrm{Na}^{+} / \mathrm{Ca}^{2+}$ exchanger. The GABA-dependent $\mathrm{Ca}^{2+}$ increase drives ATP/adenosine release that diffuses to excitatory presynaptic terminals, where it inhibits glutamate release and regulates heterosynaptic depression (Boddum et al., 2016).
Cxs have also been implicated in neuroglial communication modulation by gliotransmitter release, impacting pre- and postsynaptic terminals. In prefrontal cortex (PFC), inhibition of $\mathrm{Cx} 43 \mathrm{HCs}$ by mimetic peptide Gap26 reduces the amplitude of postsynaptic excitatory currents mediated by NMDA receptors and impairs long-term potentiation (LTP) induced by high-frequency stimulation (Meunier et al., 2017). In this area, astrocytic $\left[\mathrm{Ca}^{2+}\right] \mathrm{i}$ raised by an influx of this ion through Cx43 HCs stimulates D-serine release, a gliotransmitter and co-agonist of NMDA receptors. Hence, D-serine released from astrocytes also through $\mathrm{Cx} 43 \mathrm{HCs}$ in conjunction with glutamate induces NMDA receptor activation, a process that is important for AMPA receptor traffic and stability during LTP (Meunier et al., 2017). These results strongly suggest Cx43 HCs activity in astrocytes is a critical postsynaptic determinant of LTP in the PFC, by regulating NMDA receptor co-activation site occupancy by D-Serine (Henneberger et al., 2010). This effect is apparently independent of astrocyte intercellular communication achieved through GJ channels. Studies using $\mathrm{Cx} 43$ conditional knockout mice have revealed a presynaptic regulation of basal excitatory synaptic transmission by $\mathrm{Cx}$ (Chever et al., 2014a,b). Deletion of Cx43 in astrocytes induces a decrease in basal excitatory synaptic transmission that is coupled to changes in cell volume, without affecting intrinsic and passive membrane properties of astrocytes and activation of NMDA and AMPA receptors in neurons. Although the mechanism is still unclear, this deficiency in synaptic transmission may be explained by $\mathrm{Cx}$ metabolic coupling role between astrocytes and neurons. Astrocyte metabolites such as glutamate, glucose, lactate and glutamine permeate through $\mathrm{Cx} 43 \mathrm{HCs}$ and are taken up by neurons to supply metabolic substrates. It also provides glutamate precursors to refill synaptic vesicles that support excitatory synaptic transmission. Consequently, the observed decrease on basal synaptic excitatory neurotransmission in $\mathrm{Cx} 43 \mathrm{KO}$ mice, can be the result of a glutamate diffusion impartment in astrocytes that lead to glutamine-glutamate cycle failure, operating between astrocytes and neurons (more details in the following section; Bak et al., 2006; Chever et al., 2014b). This cycle is essential for neurons to provide glutamine utilized for glutamate and GABA synthesis at the presynaptic terminal.

Astrocytes like neurons have complex morphologies characterized by the presence of processes that protrude into synapses. For synapses to work efficiently, astrocytes and neurons should have developed mechanisms controlling astrocytic processes extension into synapses, limiting astrocyte territories in the synaptic cleft, which is essential to re-uptake neurotransmitters without affecting synaptic transmission. Cx30 knockout mice show a decreased excitatory neurotransmission and impaired synaptic plasticity (Pannasch et al., 2014). These deficiencies are accompanied by low glutamate levels, increased currents throughout GLT transporters, and significant changes in astrocytic processes extension that insert more in-depth into the synaptic cleft. The observed reduction in synaptic strength in Cx30 KO mice can be the result of an increase in glutamate clearance. This novel role of $\mathrm{Cx} 30$ is independent of its channel function, 
but it is determined by its C-terminal domain interaction with cellular elements that regulate adhesion and migration processes, limiting the extension of astrocytes in the synapse (Pannasch et al., 2014). Similar results have been found in $\mathrm{Cx} 30^{-/-} \mathrm{Cx} 43^{-/-}$mice, in which Cxs absence leads to glutamate and $\mathrm{K}^{+}$ions clearance impairments, changes in astrocyte volume, and increases in glutamate and GABA release. To maintain a regular firing without gain modifications, due to abnormal glutamate and GABA levels, Cxs alter AMPA receptor expression in the postsynaptic terminal, inducing homeostatic plasticity (Pannasch et al., 2011). Therefore, the syncytial network established by astrocytes through Cx30 and Cx43 expression is essential for regulating basal synaptic transmission and plasticity by mechanisms that involve neuronal excitability ( $\mathrm{K}^{+}$buffering), neurotransmitter release and uptake (Glutamate and GABA), and receptor stability (homeostatic plasticity).

\section{ASTROCYTIC $\left[\mathrm{Ca}^{2+}\right] \mathrm{I}$ INCREASE IS COUPLED WITH A GLIOTRANSMITTER RELEASE THROUGH Cx HCs}

Binding of synaptic neurotransmitters to high-affinity $G$ proteincoupled receptors (GPCRs) in astrocytes starts a cascade of events that stimulate membrane phospholipid hydrolysis to inositol triphosphate $\left(\mathrm{IP}_{3}\right)$ and diacylglycerol (DAG; Volterra and Meldolesi, 2005; De Pittà et al., 2009). Diffusion of $\mathrm{IP}_{3}$ then induces $\mathrm{Ca}^{2+}$ efflux from the endoplasmic reticulum, raising astrocyte $\mathrm{Ca}^{2+}$ concentration that drives gliotransmitter release. Although mechanisms that associate $\left[\mathrm{Ca}^{2+}\right]$ i increases to gliotransmitter release in astrocytes have not been fully established, is believed these bioactive molecules are released by $\mathrm{Ca}^{2+}$-dependent vesicular exocytosis (as in neurons), and non-dependent vesicular release. Astrocytes express Synaptotagmin IV (a $\mathrm{Ca}^{2+}$ sensor expressed in synaptic vesicles) and SNARE type proteins. Synaptotagmin IV knockdown experiments have revealed this $\mathrm{Ca}^{2+}$ sensor is involved in glutamate release from astrocytes (Zhang et al., 2004). Similarly, astrocytes express synaptobrevin II and cellubrevin, two proteins of the SNARE complex that regulate glutamate and neuropeptide-Y release. Remarkably, these two gliotransmitters are not only packed in different vesicles, but they also exert different effects in synaptic transmission (Schwarz et al., 2017).

Non-vesicular gliotransmitter release is achieved through reversible uptake carriers (Rossi et al., 2000), volume-activated anion channels (Rudkouskaya et al., 2008), purinergic receptors (Suadicani et al., 2006), and Cx and pannexin HCs (Panx HCs; Jiang et al., 2011; Orellana et al., 2011). The mechanisms by which astrocyte intracellular $\mathrm{Ca}^{2+}$ changes are coupled to gliotransmitters release through $\mathrm{Cx}$ and Panx $\mathrm{HCs}$ are not fully understood. However, recent findings suggest $\mathrm{Ca}^{2+}$ levels regulate $\mathrm{Cx} \mathrm{HCs}$ opening probability. For example, Cx32 HCs have a $\mathrm{Ca}^{2+}$ binding site rich in aspartate residues that sense and decode $\left[\mathrm{Ca}^{2+}\right]$ e changes in channel gating (Gómez-Hernández et al., 2003). Moreover, Cx32 HC opening is triggered by changes in $\left[\mathrm{Ca}^{2+}\right] \mathrm{i}$ in the range of $200-1000 \mathrm{nM}$. The presence of calmodulin binding sites in Cx32 and Cx43 HCs and the fact that calmodulin inhibitors block connexin-ATP release, suggest this $\mathrm{Ca}^{2+}$ sensor is part of the $\mathrm{Ca}^{2+}$ pathway utilized to regulate Cx HCs gating (De Vuyst et al., 2006; Lurtz and Louis, 2007).

Other studies using mouse epidermal cells have determined GJ intracellular communication is mediated by a $\mathrm{Ca}^{2+}$-dependent regulation of E-cadherin cell adhesion molecule (Jongen et al., 1991). Although this mechanism has not been evaluated in astrocytes, the above results suggest interactions between Cxs and cell adhesion molecules mediated by $\mathrm{Ca}^{2+}$ increases can be essential for astrocyte syncytium stability during neuronal activity.

Paracrine ATP action can complicate $\mathrm{Ca}^{2+}$ signaling in astrocytes. Extracellular ATP signaling is crucial in the CNS, and its interaction with P2-purinoceptors (P2Rs) has critical neuromodulator functions (Tozaki-Saitoh et al., 2011; Illes et al., 2012). P2YRs are metabotropic receptors coupled with different G-proteins that mediate phospholipase C (PLC) signaling, associating $\mathrm{IP}_{3}$ diffusion to $\mathrm{Ca}^{2+}$ mobilization via GJs (Cotrina et al., 1998; Illes et al., 2012). Hence, ATP- $\mathrm{Ca}^{2+}$ mobilization in astrocytes works as a feedback mechanism, regulating $\mathrm{Ca}^{2+}$ diffusion in astrocytes that allow local synaptic transmission to influence synaptic activity over a large population of neurons.

\section{METABOLIC COUPLING BETWEEN NEURONS AND GLIAL CELLS}

Astrocyte $\left[\mathrm{Ca}^{2+}\right] \mathrm{i}$ increase is also used as a signal to trigger metabolic linkage between neural activity and energy demands to sustain neuronal firing. Coupling is achieved by releasing messengers, such as arachidonic acid (AA) that regulates blood vessel tone or by increasing glucose uptake from blood (Bazargani and Attwell, 2016). This energetic coupling between astrocytes and neurons is also achieved by regulating biosynthesis, degradation and uptake of excitatory and inhibitory neurotransmitters (Figure 3).

Astrocytes express transporters that mediate glucose uptake from adjacent cerebral microvessels through glucose type 1 transporters (GLUT1; Rouach et al., 2008). Glucose is stored as glycogen and then is partially oxidized through the glycolysis pathway to produce pyruvate. However, during intense neuronal activity astrocytes reduce pyruvate to lactate and export this energetic substrate through monocarboxylate transporters 1 or 4 (MCT1-4) to the synaptic cleft, which can be taken-up by neurons via MCT2 transporters. This coupling between cells is known as astrocyte-neuron lactate shuttle (ANLS) hypothesis (Pellerin and Magistretti, 1994). Additionally, astrocytes and neurons can completely oxidize pyruvate in the Krebs cycle (KC). Neuronal aerobic glucose catabolism leads to a net synthesis of alpha-ketoglutarate $(\alpha-K G)$, catalyzed either by glutamate dehydrogenase (GDH) or an amino acid aminotransferase (AAT; Figure 3). As a result, glutamate can be synthesized and used for excitatory synaptic transmission or decarboxylated by a glutamic acid decarboxylase (GAD 65/67) for GABA synthesis in inhibitory presynaptic terminals (Walls et al., 2010, 2011). 


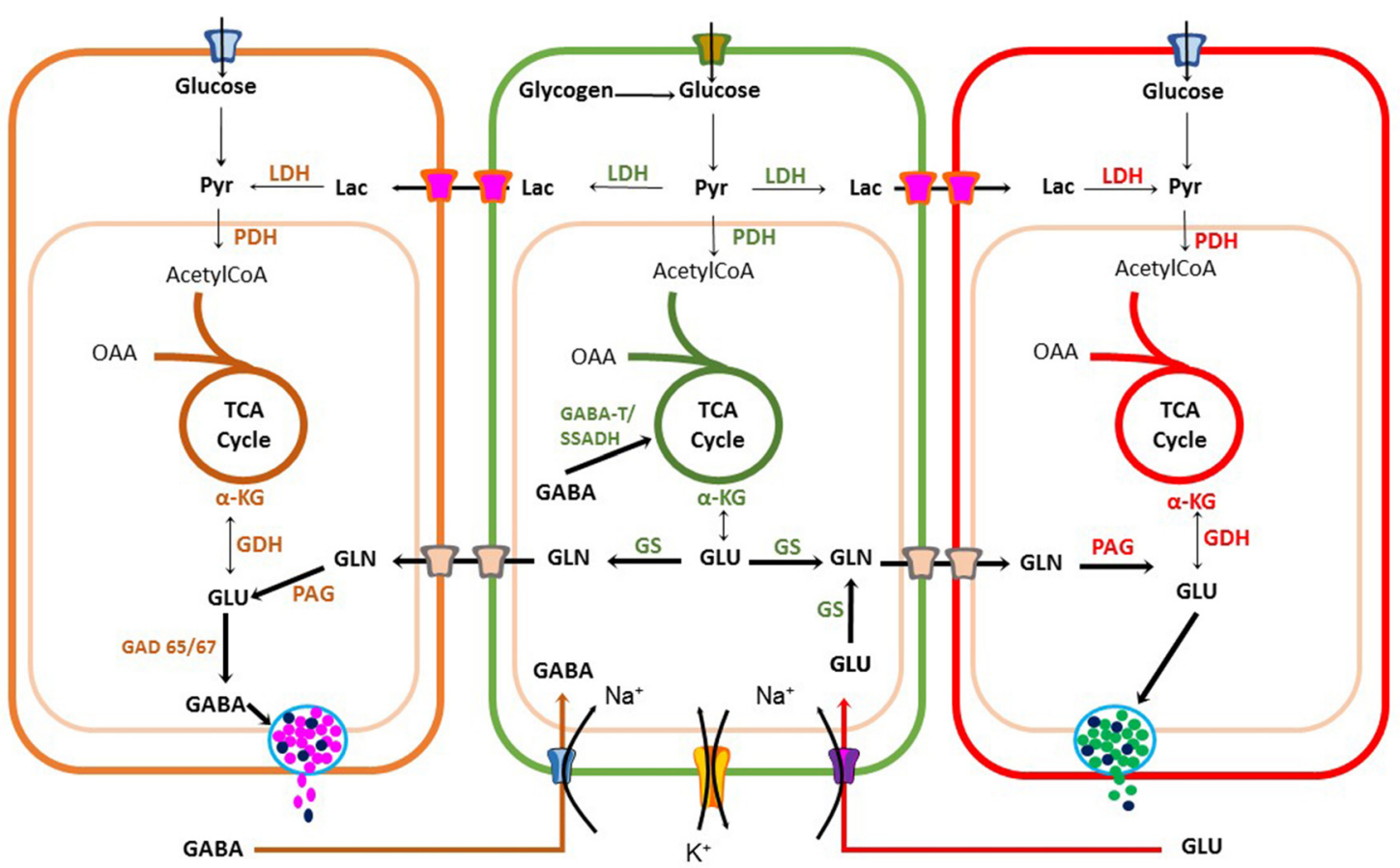

FIGURE 3 | Metabolic coupling between neurons and astrocytes during synaptic transmission. Glutamatergic and GABAergic neurons have an oxidative metabolism that depends mainly on glucose. Neurons use energy for restoring the ionic balance after action potential firing and for neurotransmitter synthesis. Astrocytes also play a significant role in recycling and inactivation of gamma-aminobutyric acid (GABA) and glutamate neurotransmitters through glutamate-GABA-glutamine cycle, also important for ammonium ion buffering. Astrocytes can also produce lactate upon increase in neuronal activity to sustain high firing rate. Pyr, Pyruvate; LDH, Lactate Dehydrogenase; OAA, Oxaloacetic acid; TCA, tricarboxylic cycle acid; aKG, alpha-ketoglutaric acid; GDH, Glutamate dehydrogenase; GLN, Glutamine; PAG, Phosphate-activated glutaminase; GAD, glutamic acid decarboxylase; GS, Glutamine Synthetase.

A family of five glutamate transporters, called GLAST, GLT-1, EAAC1, EAAT4 and EAAT5 (also known as EAAT1-5), are responsible for maintaining extracellular glutamate concentrations within a range that permits normal excitatory neurotransmission (Tzingounis and Wadiche, 2007). Astrocytes, transform glutamate to glutamine, through a reaction catalyzed by glutamine synthetases (GSs) with an expenditure of 1 ATP (Krebs, 1935). Then, glutamine is released into the extracellular milieu and is taken-up by neurons to synthesize glutamate by a phosphate-activated glutaminase (PAG; Krebs, 1935). Use of glutamate and GABA for glutamine biosynthesis by astrocytes gives origin to the GlutamateGABA-Glutamine cycle operating between glutamatergic and GABAergic tripartite synapsis (Bak et al., 2006; Figure 3). Therefore, the coupling between excitation and inhibition is a crucial condition required for normal neurotransmission. Synthesis and re-uptake of both glutamate and GABA demand neuroglia compartmentation. Glutamate-GABA-Glutamine cycle by maintaining low neurotransmitter concentrations in the synaptic cleft protects neurons against glutamate excitotoxicity. However, it is an expensive transport process requiring high ATP levels. Each neurotransmitter molecule is co-transported with three sodium ions $\left(\mathrm{Na}^{+}\right)$, requiring $\mathrm{Na}^{+} / \mathrm{K}^{+}$ATPase activity. Furthermore, in astrocytes, this coupling demands glucose utilization and oxidation (Hertz, 2013; Schousboe et al., 2013a). Hence, to sustain significant glutamate removal from the synaptic cleft, astrocytes must produce enough ATP from different energetic substrates. Another essential function of the Glutamate-GABA-Glutamine cycle is ammonium $\left(\mathrm{NH}_{4}{ }^{+}\right)$ transport (Figure 3), which is potentially toxic to neurons and it must be taken up by the surrounding astrocytes. This is a clear example of compartmentation between neurons and astrocytes (Hertz, 2013; Schousboe et al., 2013a,b). Synaptic transmission and its regulation by gliotransmitters is a demanding energy process. It requires metabolic coupling to provide energetic substrates to sustain ATP production for neurotransmitter uptake, ion pumps and guarantee the necessary precursors replenishment for neurotransmitter synthesis to maintain synaptic transmission.

\section{ROLE OF CONNEXINS IN CNS DISEASES}

As discussed above, astrocytic $\mathrm{Ca}^{2+}$-dependent signaling pathways are activated in healthy brain tissue. However, dramatic changes in astrocytic $\left[\mathrm{Ca}^{2+}\right] \mathrm{i}$ transient frequencies, duration and amplitude have been reported in epilepsy (Ding et al., 2007), Alzheimer's disease (AD; Kuchibhotla et al., 2009), and stroke (Rakers et al., 2017). These observations suggest changes occurring in reactive astrocytes could regulate liberation of gliotransmitters, instead of synchronizing normal metabolic coupling that could exacerbate injuries or lead to neurodegeneration. Some authors have proposed these events 
could be a consequence of a rapid response, seconds to minutes, part of a serial of excitotoxic events that are common to these diseases (Agulhon et al., 2012). For instance, damaged neurons after ischemia decline their aerobic metabolism, decrease ATP production, and accumulate toxic ions and molecules, such as $\mathrm{Ca}^{2+}, \mathrm{K}^{+}$, radical oxygen species (ROS), and nitric oxide (NO). These toxic molecular species can be released through HCs and propagated from damaged cells to healthy cells through GJs (Takeuchi and Suzumura, 2014). Under these conditions, those signals can activate microglia and astrocytes, inducing the release of pro-inflammatory cytokines and chemokines (Orellana et al., 2009). These inflammatory mediators can exert a modulatory effect on astrocyte physiology, causing alteration on neuronal functions that affect mood, behavior and cognitive abilities (Sofroniew, 2014). At the cellular level, pro-inflammatory cytokines reduce the intercellular communication mediated by GJs and increase $\mathrm{Cx}$ HCs activity, leading to an increase in astrocytic ATP and glutamate release (Retamal et al., 2007; Orellana et al., 2011). Under this inflammatory scenario, astrocytes not only produce, but they also respond to molecules such as tumor necrosis factor $\alpha(\mathrm{TNF} \alpha)$, transforming growth factor $\beta(\mathrm{TGF} \beta)$, IL1 $\beta$, IL6, interferon $\gamma$ (IFN $\gamma)$, CCL12, glial derived neurotrophic factor (GDNF), and proteins, such as fibrinogen, thrombin and endothelin-1 (Hamby et al., 2012; Sofroniew, 2014). Other molecules, such as prostaglandins (PG), NO and AA are produced by astrocytes and can induce deleterious effects in neurons (Avila-Muñoz and Arias, 2014). Neurodegenerative diseases like Alzheimer, Parkinson and other pathological conditions, such as ischemia and traumatic brain injury (TBI) will be discussed in the context of $\mathrm{Cx}$ HCs activity in response to injury.

\section{Cxs IN ALZHEIMER'S DISEASE}

$\mathrm{AD}$ is the leading prevalent dementia in the world and is considered a neurodegenerative condition characterized by a decline of the cognitive function related to a progressive cortical neuronal loss. Although the primary risk factor is aging, at the molecular level, $\mathrm{AD}$ has been associated with extracellular accumulation of beta-amyloid peptide $(\mathrm{A} \beta)$ and intracellular neurofibrillary tangles of hyperphosphorylated Tau protein, loss of synaptic connections, and oxidative stress increase leading to neuronal death (Querfurth and LaFerla, 2010). A classical $\mathrm{AD}$ study in human brains found increased $\mathrm{Cx} 43$ expression in reactive astrocytes near amyloid plaques, activated microglia, and neurons (Nagy et al., 1996). Other studies using two murine models of $\mathrm{AD}$ for $\beta$-amyloid precursor protein (APP) and presenilin 1 (PS1) have found Cx43, and Cx30 expression was increased in activated astrocytes that were associated with amyloid plaques in the pyramidal cell layer of the hippocampus at 6 months (Mei et al., 2010). Human brain samples with a diagnosis of AD and Parkinson's disease (PD) have also identified increased $\mathrm{Cx} 43$ expression associated with astrocytosis, a process that correlates with the progression of both diseases (Kim I. S. et al., 2016). Moreover, studies evaluating APP/PS1 mice have shown $\mathrm{Cx} 43$ overexpression that correlates with gliotransmitter increments release, such as ATP, glutamate, and $\mathrm{Ca}^{2+}$, increase in oxidative stress and exacerbated neuronal damage (Yi et al., 2016). Nowadays it is a matter of debate if the increased Cxs expression in $\mathrm{AD}$ is part of an adaptive molecular mechanism to restore neuroglia homeostasis communication, or instead, it is an aberrant protein expression that exacerbates cellular conditions and microenvironment favoring disease progress.

\section{CXS IN PARKINSON'S DISEASE}

$\mathrm{PD}$ is the second neurodegenerative disorder worldwide. Its main feature is the loss of dopaminergic neurons in the substance nigra pars compacta resulting in decreased dopamine levels, increased oxidative stress, and mitochondrial dysfunction in the striatum. As consequence patients display bradykinesia, rigidity and tremor (Lees et al., 2009). A recent study has shown that overexpression of $\mathrm{Cx} 43$ protects SH-SY5Y neuroblastoma cells, against mitochondrial-induced apoptosis by regulating mitochondrial permeability transition pore closing, preservation of mitochondrial membrane potential, and decreasing cytochrome C release (Kim I. S. et al., 2016). In vitro and in vivo studies using rotenone-induced models have shown increased expression of $\mathrm{Cx} 43$ and its phosphorylated form (Kawasaki et al., 2009). However, these changes in Cx43expression levels do not correlate with neuronal survival. Phosphorylation of $\mathrm{Cx}$ has been associated with functional GJs assembly, therefore in PD Cx43 phosphorylation could be related to mechanisms that reestablish normal synaptic function and promote neuronal survival.

Other studies using SH SY5Y cells that overexpress wild-type $\alpha$-synuclein demonstrated those cells were more susceptible to hydrogen peroxide and 6-hydroxydopamine. This increased susceptibility was mediated by a molecular interaction between $\mathrm{Cx} 32$ and $\alpha$-synuclein, suggesting that modulatory mechanisms of GJs can be essential to high sensitivity to neuronal toxins (Sung et al., 2007).

\section{CXS IN ACUTE CEREBRAL ISCHEMIA AND TRAUMATIC BRAIN INJURY (TBI)}

Anomalous functioning of $\mathrm{Cx} 43$ after ischemic insults causes apoptotic cell death. In a cerebral rat model of ischemia, clamping both arteries increased expression of heteromeric $\mathrm{Cx} 40 / \mathrm{Cx} 43$ connexons and $\mathrm{Cx} 43$ phosphorylated form. This increase in $\mathrm{Cx}$ levels was mediated after ischemia by extracellular signal-regulated kinase (ERK) activation (Chen et al., 2017), suggesting heteromeric $\mathrm{Cx}$ complexes contribute to brain damage.

In neonatal rat models of hypoxia/ischemia (HI), $\mathrm{Cx} 43$ expression increased, and pre-treatment with Gap26 and Gap27 peptides powerfully decreased cerebral infarct volume and improved muscle strength, motor coordination and spatial memory skills. Furthermore, Gap26 treatment reduced $\mathrm{Cx} 43$ expression and astrocytosis after $\mathrm{HI}$ insult, suggesting $\mathrm{Cx} 43$ could be considered a therapeutic target ischemic or hypoxic disease treatment ( $\mathrm{Li}$ et al., 2015). This hypothesis is further supported by recent findings in TBI that have shown 
increased levels of phosphorylated $\mathrm{Cx} 43$ raised exosome marker expression and release, which can be activated as protective mechanisms, improving LTP inhibited in TBI (Chen et al., 2018).

\section{FINAL CONSIDERATIONS}

During neural activity interglial communication regulates neurotransmitter and $\mathrm{K}^{+}$buffering by potentiating astrocytic syncytium function. This global integration by astrocytes is essential for functional and metabolic coupling of astrocytes and neurons. Under pathological conditions; although there is an increase in Cxs expression that can stimulate the syncytium ensemble, this process is impaired by a shift towards HC activity. This alteration is mediated by several pro-inflammatory cytokines (TNF- $\alpha$ and IL- $\beta$ ), $\mathrm{Ca}^{2+}$, and metabolic changes that stimulate the probability of pore opening (Retamal et al., 2007; Froger et al., 2010; Kim Y. et al., 2016). This evidence reveals a rise in $\mathrm{Cx}$ HCs activity can be a shared phenomenon in neurodegenerative diseases, ischemia and trauma. Additionally, a hallmark in the onset of these diseases could be a decrease in $\mathrm{Cx}$ docking required for functional GJs generation. Therefore, search for molecules that can regulate

\section{REFERENCES}

Agulhon, C., Sun, M. Y., Murphy, T., Myers, T., Lauderdale, K., and Fiacco, T. A. (2012). Calcium signaling and gliotransmission in normal vs. Reactive astrocytes. Front. Pharmacol. 3:139. doi: 10.3389/fphar.2012.00139

Avila-Muñoz, E., and Arias, C. (2014). When astrocytes become harmful: functional and inflammatory responses that contribute to Alzheimer's disease. Ageing Res. Rev. 18, 29-40. doi: 10.1016/j.arr.2014.07.004

Bak, L. K., Schousboe, A., and Waagepetersen, H. S. (2006). The glutamate/GABAglutamine cycle: aspects of transport, neurotransmitter homeostasis and ammonia transfer. J. Neurochem. 98, 641-653. doi: 10.1111/j.1471-4159.2006. 03913.x

Barres, B. A. (2008). The mystery and magic of glia: a perspective on their roles in health and disease. Neuron 60, 430-440. doi: 10.1016/j.neuron.2008. 10.013

Bazargani, N., and Attwell, D. (2016). Astrocyte calcium signaling: the third wave. Nat. Neurosci. 19, 182-189. doi: 10.1038/nn.4201

Ben Haim, L., and Rowitch, D. H. (2017). Functional diversity of astrocytes in neural circuit regulation. Nat. Rev. Neurosci. 18, 31-41. doi: 10.1038/nrn. 2016.159

Bezzi, P., and Volterra, A. (2001). A neuron-glia signalling network in the active brain. Curr. Opin. Neurobiol. 11, 387-394. doi: 10.1016/s0959-4388(00) 00223-3

Boddum, K., Jensen, T. P., Magloire, V., Kristiansen, U., Rusakov, D. A., Pavlov, I., et al. (2016). Astrocytic GABA transporter activity modulates excitatory neurotransmission. Nat. Commun. 7:13572. doi: 10.1038/ncomms13572

Bowser, D. N., and Khakh, B. S. (2007). Vesicular ATP is the predominant cause of intercellular calcium waves in astrocytes. J. Gen. Physiol. 129, 485-491. doi: 10.1085/jgp.200709780

Chen, W., Feng, J., and Tong, W. (2017). Phosphorylation of astrocytic connexin 43 by ERK1/2 impairs blood-brain barrier in acute cerebral ischemia. Cell Biosci. 7:43. doi: 10.1186/s13578-017-0170-6

Chen, W., Guo, Y., Yang, W., Chen, L., Ren, D., Wu, C., et al. (2018). Phosphorylation of connexin 43 induced by traumatic brain injury promotes exosome release. J. Neurophysiol. 119, 305-311. doi: 10.1152/jn. 00654.2017

Chever, O., Lee, C. Y., and Rouach, N. (2014a). Astroglial connexin43 hemichannels tune basal excitatory synaptic transmission. J. Neurosci. 34, 11228-11232. doi: 10.1523/JNEUROSCI.0015-14.2014
HC pore opening and connexon ensemble can have therapeutic potential.

\section{AUTHOR CONTRIBUTIONS}

LCM and AVR wrote the article and drew the figures. J-JS wrote the article and SLA conceptualized and wrote the article.

\section{FUNDING}

This work was funded by Pontificia Universidad Javeriana Vicerrectoría de investigación Project No. 00005879: Evaluación de la capacidad de diferentes tipos de flavonoides (flavonoles, flavonas y flavononas y sus glucósidos) para modular la activación astrocítica in vitro inducida por Lipopolisacáridos.

\section{ACKNOWLEDGMENTS}

The authors thank Pontificia Universidad Javeriana for financial support. The authors want to express their gratitude to Dr. Juan Carlos Sáez for the critical reading of this manuscript.

Chever, O., Pannasch, U., Ezan, P., and Rouach, N. (2014b). Astroglial connexin 43 sustains glutamatergic synaptic efficacy. Philos. Trans. R. Soc. Lond. B Biol. Sci. 369:20130596. doi: 10.1098/rstb.2013.0596

Cornell-Bell, A. H., Finkbeiner, S. M., Cooper, M. S., and Smith, S. J. (1990). Glutamate induces calcium waves in cultured astrocytes: long-range glial signaling. Science 247, 470-473. doi: 10.1126/science.1967852

Cotrina, M. L., Lin, J. H., Alves-Rodrigues, A., Liu, S., Li, J., Azmi-Ghadimi, H., et al. (1998). Connexins regulate calcium signaling by controlling ATP release. Proc. Natl. Acad. Sci. U S A 95, 15735-15740. doi: 10.1073/pnas.95.26. 15735

Dallérac, G., Chever, O., and Rouach, N. (2013). How do astrocytes shape synaptic transmission? Insights from electrophysiology. Front. Cell. Neurosci. 7:159. doi: 10.3389/fncel.2013.00159

De Bock, M., Leybaert, L., and Giaume, C. (2017). Connexin channels at the glio-vascular interface: gatekeepers of the brain. Neurochem. Res. 42, 2519-2536. doi: 10.1007/s11064-017-2313-x

De Pittà, M., Goldberg, M., Volman, V., Berry, H., and Ben-Jacob, E. (2009). Glutamate regulation of calcium and IP3 oscillating and pulsating dynamics in astrocytes. J. Biol. Phys. 35, 383-411. doi: 10.1007/s10867-009 $-9155-y$

De Vuyst, E., Decrock, E., Cabooter, L., Dubyak, G. R., Naus, C. C., Evans, W. H., et al. (2006). Intracellular calcium changes trigger connexin 32 hemichannel opening. EMBO J. 25, 34-44. doi: 10.1038/sj.emboj.7600908

Ding, S., Fellin, T., Zhu, Y., Lee, S. Y., Auberson, Y. P., Meaney, D. F., et al. (2007). Enhanced astrocytic $\mathrm{Ca}^{2+}$ signals contribute to neuronal excitotoxicity after status epilepticus. J. Neurosci. 27, 10674-10684. doi: 10.1523/JNEUROSCI. 2001-07.2007

Duffy, S., and MacVicar, B. A. (1995). Adrenergic calcium signaling in astrocyte networks within the hippocampal slice. J. Neurosci. 15, 5535-5550.

Elias, L. A., Turmaine, M., Parnavelas, J. G., and Kriegstein, A. R. (2010). Connexin 43 mediates the tangential to radial migratory switch in ventrally derived cortical interneurons. J. Neurosci. 30, 7072-7077. doi: 10.1523/JNEUROSCI. 5728-09.2010

Fiacco, T. A., Agulhon, C., Taves, S. R., Petravicz, J., Casper, K. B., Dong, X., et al. (2007). Selective stimulation of astrocyte calcium in situ does not affect neuronal excitatory synaptic activity. Neuron 54, 611-626. doi: 10.1016/j. neuron.2007.04.032

Froger, N., Orellana, J. A., Calvo, C. F., Amigou, E., Kozoriz, M. G., Naus, C. C., et al. (2010). Inhibition of cytokine-induced connexin 43 hemichannel activity 
in astrocytes is neuroprotective. Mol. Cell. Neurosci. 45, 37-46. doi: 10.1016/j. mcn.2010.05.007

Gómez-Hernández, J. M., de Miguel, M., Larrosa, B., González, D., and Barrio, L. C. (2003). Molecular basis of calcium regulation in connexin-32 hemichannels. Proc. Natl. Acad. Sci. U S A 100, 16030-16035. doi: 10.1073/ pnas. 2530348100

Hamby, M. E., Coppola, G., Ao, Y., Geschwind, D. H., Khakh, B. S., and Sofroniew, M. V. (2012). Inflammatory mediators alter the astrocyte transcriptome and calcium signaling elicited by multiple G-proteincoupled receptors. J. Neurosci. 32, 14489-14510. doi: 10.1523/JNEUROSCI. 1256-12.2012

Henneberger, C., Papouin, T., Oliet, S. H., and Rusakov, D. A. (2010). Longterm potentiation depends on release of D-serine from astrocytes. Nature 463, 232-236. doi: 10.1038/nature08673

Hertz, L. (2013). The glutamate-glutamine (GABA) cycle: importance of late postnatal development and potential reciprocal interactions between biosynthesis and degradation. Front. Endocrinol. 4:59. doi: 10.3389/fendo.2013. 00059

Hu, B., Sun, S. G., and Tong, E. T. (2004). NMDA and AMPA receptors mediate intracellular calcium increase in rat cortical astrocytes. Acta Pharmacol. Sin. 25, $714-720$.

Illes, P., Verkhratsky, A., Burnstock, G., and Franke, H. (2012). P2X receptors and their roles in astroglia in the central and peripheral nervous system. Neuroscientist 18, 422-438. doi: 10.1177/1073858411418524

Jiang, S., Yuan, H., Duan, L., Cao, R., Gao, B., Xiong, Y. F., et al. (2011). Glutamate release through connexin 43 by cultured astrocytes in a stimulated hypertonicity model. Brain Res. 1392, 8-15. doi: 10.1016/j.brainres.2011. 03.056

Jongen, W. M., Fitzgerald, D. J., Asamoto, M., Piccoli, C., Slaga, T. J., Gros, D., et al. (1991). Regulation of connexin 43-mediated gap junctional intercellular communication by $\mathrm{Ca}^{2+}$ in mouse epidermal cells is controlled by E-cadherin. J. Cell Biol. 114, 545-555. doi: 10.1083/jcb.114.3.545

Kang, J., Jiang, L., Goldman, S. A., and Nedergaard, M. (1998). Astrocyte-mediated potentiation of inhibitory synaptic transmission. Nat. Neurosci. 1, 683-692. doi: $10.1038 / 3684$

Kawasaki, A., Hayashi, T., Nakachi, K., Trosko, J. E., Sugihara, K., Kotake, Y., et al. (2009). Modulation of connexin 43 in rotenone-induced model of Parkinson's disease. Neuroscience 160, 61-68. doi: 10.1016/j.neuroscience.2009. 01.080

Kim, Y., Davidson, J. O., Gunn, K. C., Phillips, A. R., Green, C. R., and Gunn, A. J. (2016). Role of hemichannels in CNS inflammation and the inflammasome pathway. Adv. Protein Chem. Struct. Biol. 104, 1-37. doi: 10.1016/bs.apcsb. 2015.12.001

Kim, I. S., Ganesan, P., and Choi, D. K. (2016). Cx43 mediates resistance against $\mathrm{MPP}^{+}$-induced apoptosis in SH-SY5Y neuroblastoma cells via modulating the mitochondrial apoptosis pathway. Int. J. Mol. Sci. 17:E1819. doi: 10.3390/ijms17111819

Krebs, H. A. (1935). Metabolism of amino-acids: the synthesis of glutamine from glutamic acid and ammonia and the enzymic hydrolysis of glutamine in animal tissues. Biochem. J. 29, 1951-1969. doi: 10.1042/bj0291951

Kuchibhotla, K. V., Lattarulo, C. R., Hyman, B. T., and Bacskai, B. J. (2009). Synchronous hyperactivity and intercellular calcium waves in astrocytes in Alzheimer mice. Science 323, 1211-1215. doi: 10.1126/science. 1169096

Lapato, A. S., Szu, J. I., Hasselmann, J. P. C., Khalaj, A. J., Binder, D. K., and TiwariWoodruff, S. K. (2017). Chronic demyelination-induced seizures. Neuroscience 346, 409-422. doi: 10.1016/j.neuroscience.2017.01.035

Lapato, A. S., and Tiwari-Woodruff, S. K. (2018). Connexins and pannexins: at the junction of neuro-glial homeostasis and disease. J. Neurosci. Res. 96, 31-44. doi: $10.1002 /$ jnr. 24088

Lees, A. J., Hardy, J., and Revesz, T. (2009). Parkinson's disease. Lancet 373, 2055-2066. doi: 10.1016/S0140-6736(09)60492-X

Li, X., Zhao, H., Tan, X., Kostrzewa, R. M., Du, G., Chen, Y., et al. (2015). Inhibition of connexin 43 improves functional recovery after ischemic brain injury in neonatal rats. Glia 63, 1553-1567. doi: 10.1002/glia. 22826

Liebmann, M., Stahr, A., Guenther, M., Witte, O. W., and Frahm, C. (2013). Astrocytic Cx43 and Cx30 differentially modulate adult neurogenesis in mice. Neurosci. Lett. 545, 40-45. doi: 10.1016/j.neulet.2013.04.013
Liebner, S., Czupalla, C. J., and Wolburg, H. (2011). Current concepts of bloodbrain barrier development. Int. J. Dev. Biol. 55, 467-476. doi: 10.1387/ijdb. 103224 sl

Lurtz, M. M., and Louis, C. F. (2007). Intracellular calcium regulation of connexin43. Am. J. Physiol. Cell Physiol. 293, C1806-C1813. doi: 10.1152/ajpcell.00630.2006

Magnotti, L. M., Goodenough, D. A., and Paul, D. L. (2011). Deletion of oligodendrocyte $\mathrm{Cx} 32$ and astrocyte $\mathrm{Cx} 43$ causes white matter vacuolation, astrocyte loss and early mortality. Glia 59, 1064-1074. doi: 10.1002/glia.21179

Márquez-Rosado, L., Solan, J. L., Dunn, C. A., Norris, R. P., and Lampe, P. D. (2012). Connexin43 phosphorylation in brain, cardiac, endothelial and epithelial tissues. Biochim. Biophys. Acta 1818, 1985-1992. doi: 10.1016/j. bbamem.2011.07.028

Matsuuchi, L., and Naus, C. C. (2013). Gap junction proteins on the move: connexins, the cytoskeleton and migration. Biochim. Biophys. Acta 1828, 94-108. doi: 10.1016/j.bbamem.2012.05.014

Mei, X., Ezan, P., Giaume, C., and Koulakoff, A. (2010). Astroglial connexin immunoreactivity is specifically altered at $\beta$-amyloid plaques in $\beta$-amyloid precursor protein/presenilin1 mice. Neuroscience 171, 92-105. doi: 10.1016/j. neuroscience.2010.08.001

Meunier, C., Wang, N., Yi, C., Dallerac, G., Ezan, P., Koulakoff, A., et al. (2017). Contribution of astroglial Cx43 hemichannels to the modulation of glutamatergic currents by D-serine in the mouse prefrontal cortex. J. Neurosci. 37, 9064-9075. doi: 10.1523/JNEUROSCI.2204-16.2017

Nagy, J. I., Li, W., Hertzberg, E. L., and Marotta, C. A. (1996). Elevated connexin43 immunoreactivity at sites of amyloid plaques in Alzheimer's disease. Brain Res. 717, 173-178. doi: 10.1016/0006-8993(95)01526-4

Navarrete, M., and Araque, A. (2008). Endocannabinoids mediate neuronastrocyte communication. Neuron 57, 883-893. doi: 10.1016/j.neuron.2008. 01.029

Oberheim, N. A., Takano, T., Han, X., He, W., Lin, J. H., Wang, F., et al. (2009). Uniquely hominid features of adult human astrocytes. J. Neurosci. 29, 3276-3287. doi: 10.1523/JNEUROSCI.4707-08.2009

Orellana, J. A., Froger, N., Ezan, P., Jiang, J. X., Bennett, M. V., Naus, C. C., et al. (2011). ATP and glutamate released via astroglial connexin 43 hemichannels mediate neuronal death through activation of pannexin 1 hemichannels. J. Neurochem. 118, 826-840. doi: 10.1111/j.1471-4159.2011.07210.x

Orellana, J. A., Sáez, P. J., Shoji, K. F., Schalper, K. A., Palacios-Prado, N., Velarde, V., et al. (2009). Modulation of brain hemichannels and gap junction channels by pro-inflammatory agents and their possible role in neurodegeneration. Antioxid. Redox Signal. 11, 369-399. doi: 10.1089/ars. 2008.2130

Orellana, J. A., and Stehberg, J. (2014). Hemichannels: new roles in astroglial function. Front. Physiol. 5:193. doi: 10.3389/fphys.2014.00193

Pannasch, U., Freche, D., Dallérac, G., Ghézali, G., Escartin, C., Ezan, P., et al. (2014). Connexin 30 sets synaptic strength by controlling astroglial synapse invasion. Nat. Neurosci. 17, 549-558. doi: 10.1038/nn.3662

Pannasch, U., Vargová, L., Reingruber, J., Ezan, P., Holcman, D., Giaume, C., et al. (2011). Astroglial networks scale synaptic activity and plasticity. Proc. Natl. Acad. Sci. U S A 108, 8467-8472. doi: 10.1073/pnas.1016650108

Pasti, L., Volterra, A., Pozzan, T., and Carmignoto, G. (1997). Intracellular calcium oscillations in astrocytes: a highly plastic, bidirectional form of communication between neurons and astrocytes in situ. J. Neurosci. 17, 7817-7830.

Pellerin, L., and Magistretti, P. J. (1994). Glutamate uptake into astrocytes stimulates aerobic glycolysis: a mechanism coupling neuronal activity to glucose utilization. Proc. Natl. Acad. Sci. U S A 91, 10625-10629. doi: 10.1073/ pnas.91.22.10625

Perea, G., and Araque, A. (2005). Properties of synaptically evoked astrocyte calcium signal reveal synaptic information processing by astrocytes. J. Neurosci. 25, 2192-2203. doi: 10.1523/JNEUROSCI.3965-04.2005

Porter, J. T., and McCarthy, K. D. (1997). Astrocytic neurotransmitter receptors in situ and in vivo. Prog. Neurobiol. 51, 439-455. doi: 10.1016/s03010082(96)00068-8

Qi, G. J., Chen, Q., Chen, L. J., Shu, Y., Bu, L. L., Shao, X. Y., et al. (2016). Phosphorylation of connexin 43 by Cdk5 modulates neuronal migration during embryonic brain development. Mol. Neurobiol. 53, 2969-2982. doi: 10.1007/s12035-015-9190-6

Querfurth, H. W., and LaFerla, F. M. (2010). Alzheimer's disease. N. Engl. J. Med. 362, 329-344. doi: 10.1056/NEJMra0909142 
Rakers, C., Schmid, M., and Petzold, G. C. (2017). TRPV4 channels contribute to calcium transients in astrocytes and neurons during peri-infarct depolarizations in a stroke model. Glia 65, 1550-1561. doi: 10.1002/glia.23183

Ramón y Cajal, S. (1909). Histologie du Système Nerveux de l'Homme et des Vertébrés. Paris: Maloine.

Retamal, M. A., Froger, N., Palacios-Prado, N., Ezan, P., Sáez, P. J., Sáez, J. C., et al. (2007). Cx43 hemichannels and gap junction channels in astrocytes are regulated oppositely by proinflammatory cytokines released from activated microglia. J. Neurosci. 27, 13781-13792. doi: 10.1523/JNEUROSCI. 2042-07.2007

Ribak, C. E., Tong, W. M., and Brecha, N. C. (1996). GABA plasma membrane transporters, GAT-1 and GAT-3, display different distributions in the rat hippocampus. J. Comp. Neurol. 367, 595-606. doi: 10.1002/(sici)10969861(19960415)367:4<595::aid-cne9>3.0.co;2-\#

Robertson, J. M. (2013). Astrocyte domains and the three-dimensional and seamless expression of consciousness and explicit memories. Med. Hypotheses 81, 1017-1024. doi: 10.1016/j.mehy.2013.09.021

Rossi, D. J., Oshima, T., and Attwell, D. (2000). Glutamate release in severe brain ischaemia is mainly by reversed uptake. Nature 403, 316-321. doi: $10.1038 / 35002090$

Rouach, N., Koulakoff, A., Abudara, V., Willecke, K., and Giaume, C. (2008). Astroglial metabolic networks sustain hippocampal synaptic transmission. Science 322, 1551-1555. doi: 10.1126/science. 1164022

Rudkouskaya, A., Chernoguz, A., Haskew-Layton, R. E., and Mongin, A. A. (2008). Two conventional protein kinase $\mathrm{C}$ isoforms, $\alpha$ and $\beta \mathrm{I}$, are involved in the ATP-induced activation of volume-regulated anion channel and glutamate release in cultured astrocytes. J. Neurochem. 105, 2260-2270. doi: 10.1111/j. 1471-4159.2008.05312.x

Schousboe, A., Bak, L. K., Madsen, K. K., and Waagepetersen, H. S. (2013a). "Amino acid neurotransmitter synthesis and removal," in Neuroglia, 3rd Edn. eds H. Kettenmann and B. R. Ransom (New York, NY: Oxford University Press), 443-456.

Schousboe, A., Bak, L. K., and Waagepetersen, H. S. (2013b). Astrocytic control of biosynthesis and turnover of the neurotransmitters glutamate and GABA. Front. Endocrinol. 4:102. doi: 10.3389/fendo.2013.00102

Schwarz, Y., Zhao, N., Kirchhoff, F., and Bruns, D. (2017). Astrocytes control synaptic strength by two distinct $\mathrm{v}$-SNARE-dependent release pathways. Nat. Neurosci. 20, 1529-1539. doi: 10.1038/nn.4647

Shelton, M. K., and McCarthy, K. D. (1999). Mature hippocampal astrocytes exhibit functional metabotropic and ionotropic glutamate receptors in situ. Glia 26, 1-11. doi: 10.1002/(sici)1098-1136(199903)26:1<1::aid-glia1>3.0. co; $2-\mathrm{z}$

Shelton, M. K., and McCarthy, K. D. (2000). Hippocampal astrocytes exhibit $\mathrm{Ca}^{2+}$-elevating muscarinic cholinergic and histaminergic receptors in situ. J. Neurochem. 74, 555-563. doi: 10.1046/j.1471-4159.2000.740555.x

Sofroniew, M. V. (2014). Multiple roles for astrocytes as effectors of cytokines and inflammatory mediators. Neuroscientist 20, 160-172. doi: $10.1177 / 1073858413504466$

Sofroniew, M., and Vinters, H. (2010). Astrocytes: biology and pathology. Acta Neuropathol. 119, 7-35. doi: 10.1007/s00401-009-0619-8

Suadicani, S. O., Brosnan, C. F., and Scemes, E. (2006). P2X7 receptors mediate ATP release and amplification of astrocytic intercellular $\mathrm{Ca}^{2+}$ signaling. J. Neurosci. 26, 1378-1385. doi: 10.1523/jneurosci.3902-05.2006

Sung, J. Y., Lee, H. J., Jeong, E. I., Oh, Y., Park, J., Kang, K. S., et al. (2007). $\alpha$-synuclein overexpression reduces gap junctional intercellular communication in dopaminergic neuroblastoma cells. Neurosci. Lett. 416, 289-293. doi: 10.1016/j.neulet.2007.02.025
Takeuchi, H., and Suzumura, A. (2014). Gap junctions and hemichannels composed of connexins: potential therapeutic targets for neurodegenerative diseases. Front. Cell. Neurosci. 8:189. doi: 10.3389/fncel.2014. 00189

Tozaki-Saitoh, H., Tsuda, M., and Inoue, K. (2011). Role of purinergic receptors in CNS function and neuroprotection. Adv. Pharmacol. 61, 495-528. doi: 10.1016/b978-0-12-385526-8.00015-1

Tzingounis, A. V., and Wadiche, J. I. (2007). Glutamate transporters: confining runaway excitation by shaping synaptic transmission. Nat. Rev. Neurosci. 8, 935-947. doi: 10.1038/nrn2274

Verkhratsky, A., Orkand, R. K., and Kettenmann, H. (1998). Glial calcium: homeostasis and signaling function. Physiol. Rev. 78, 99-141. doi: 10.1152/physrev.1998.78.1.99

Volterra, A., Liaudet, N., and Savtchouk, I. (2014). Astrocyte $\mathrm{Ca}^{2+}$ signalling: an unexpected complexity. Nat. Rev. Neurosci. 15, 327-335. doi: 10.1038/nrn3725

Volterra, A., and Meldolesi, J. (2005). Astrocytes, from brain glue to communication elements: the revolution continues. Nat. Rev. Neurosci. 6, 626-640. doi: 10.1038/nrn1722

Walls, A. B., Eyjolfsson, E. M., Smeland, O. B., Nilsen, L. H., Schousboe, I., Schousboe, A., et al. (2011). Knockout of GAD65 has major impact on synaptic GABA synthesized from astrocyte-derived glutamine. J. Cereb. Blood Flow Metab. 31, 494-503. doi: 10.1038/jcbfm.2010.115

Walls, A. B., Nilsen, L. H., Eyjolfsson, E. M., Vestergaard, H. T., Hansen, S. L., Schousboe, A., et al. (2010). GAD65 is essential for synthesis of GABA destined for tonic inhibition regulating epileptiform activity. J. Neurochem. 115, 1398-1408. doi: 10.1111/j.1471-4159.2010.07043.x

Watanabe, M., Maemura, K., Kanbara, K., Tamayama, T., and Hayasaki, H. (2002). GABA and GABA receptors in the central nervous system and other organs. Int. Rev. Cytol. 213, 1-47. doi: 10.1016/s0074-7696(02)13011-7

White, T. W., and Bruzzone, R. (1996). Multiple connexin proteins in single intercellular channels: connexin compatibility and functional consequences. J. Bioenerg. Biomembr. 28, 339-350. doi: 10.1007/bf02110110

Yi, C., Mei, X., Ezan, P., Mato, S., Matias, I., Giaume, C., et al. (2016). Astroglial connexin 43 contributes to neuronal suffering in a mouse model of Alzheimer's disease. Cell Death Differ. 23, 1691-1701. doi: 10.1038/cdd. 2016.63

Zhang, Q., Fukuda, M., Van Bockstaele, E., Pascual, O., and Haydon, P. G. (2004). Synaptotagmin IV regulates glial glutamate release. Proc. Natl. Acad. Sci. U S A 101, 9441-9446. doi: 10.1073/pnas.0401960101

Zhang, Q., Hu, B., Sun, S., and Tong, E. (2003). Induction of increased intracellular calcium in astrocytes by glutamate through activating NMDA and AMPA receptors. J. Huazhong Univ. Sci. Technol. Med. Sci. 23, 254-257. doi: $10.1007 / \mathrm{bf} 02829506$

Conflict of Interest Statement: The authors declare that the research was conducted in the absence of any commercial or financial relationships that could be construed as a potential conflict of interest.

The reviewer JCS and handling Editor declared their shared affiliation.

Copyright (๔ 2018 Mayorquin, Rodriguez, Sutachan and Albarracin. This is an open-access article distributed under the terms of the Creative Commons Attribution License (CC BY). The use, distribution or reproduction in other forums is permitted, provided the original author(s) and the copyright owner are credited and that the original publication in this journal is cited, in accordance with accepted academic practice. No use, distribution or reproduction is permitted which does not comply with these terms. 